\title{
Impact of Public Health Education Program on the Novel Coronavirus Outbreak in the United States
}

\begin{abstract}
Enahoro Iboi ${ }^{1 *}$, Ariana Richardson ${ }^{1}$, Rachel Ruffin ${ }^{1}$, DeAndrea Ingram ${ }^{1}$, Jailyn Clark ${ }^{1}$, Jala Hawkins ${ }^{1}$, Maati McKinney ${ }^{1}$, Nianza Horne ${ }^{1}$, Reyla Ponder ${ }^{1}$, Zoe Denton ${ }^{1}$, Folashade B. Agusto ${ }^{2}$, Bismark Oduro ${ }^{3}$ and Lanre Akinyemi ${ }^{4}$

${ }^{1}$ Department of Mathematics, Spelman College, Atlanta, GA, United States, ${ }^{2}$ Department of Ecology \& Evolutionary Biology, University of Kansas, Lawrence, KS, United States, ${ }^{3}$ Department of Mathematics and Physical Sciences, California University of Pennsylvania, California, PA, United States, ${ }^{4}$ Department of Mathematics, Prairie View A\& M University, Prairie View, TX, United States
\end{abstract}

The coronavirus outbreak in the United States continues to pose a serious threat to human lives. Public health measures to slow down the spread of the virus involve using a face mask, social-distancing, and frequent hand washing. Since the beginning of the pandemic, there has been a global campaign on the use of non-pharmaceutical interventions (NPIs) to curtail the spread of the virus. However, the number of cases, mortality, and hospitalization continue to rise globally, including in the United States. We developed a mathematical model to assess the impact of a public health education program on the coronavirus outbreak in the United States. Our simulation showed the prospect of an effective public health education program in reducing both the cumulative and daily mortality of the novel coronavirus. Finally, our result suggests the need to obey public health measures as loss of willingness would increase the cumulative and daily mortality in the United States.

Keywords: COVID-19, public health education, non-pharmaceutical intervention, face mask, social distancing

\section{INTRODUCTION}

The novel coronavirus (COVID-19) pandemic caused by SARS-CoV-2 was first reported in Wuhan, China in December 2019 and later declared a pandemic by the World Health Organization (WHO) on March 11, 2020 (1-3). The emergence of the virus continues to cause devastating public health, and social-economic impact around the globe, including the United States $(4,5)$. The symptoms for COVID-19, which are similar to the common cold, though potentially more severe, include fever, cough, shortness of breath, fatigue, loss of taste or smell, sore throat, running nose, nausea, and diarrhea (6). As of December 12, 2020, there are over 71 million confirmed COVID-19 cases globally, resulting in over 1.6 million deaths (7). Within the United States, there have been over 16 million confirmed cases of coronavirus, with over 297,501 deaths (4).

The Centers for Disease Control and Prevention (CDC) on April 2, 2020, recommended the use of non-pharmaceutical interventions (NPIs) such as face masks in public (see Figure 1) and to practice social-distancing to curtail the spread of the virus $(3,5,8-11)$. Non-pharmaceutical interventions have had a long history of preventing many infectious diseases such as the pandemic Influenza, Measles, and the Ebola Virus Disease (EVD) (12-16). Actions taken in the early stage of the coronavirus outbreak by the various state governments in the United States include declaring a 

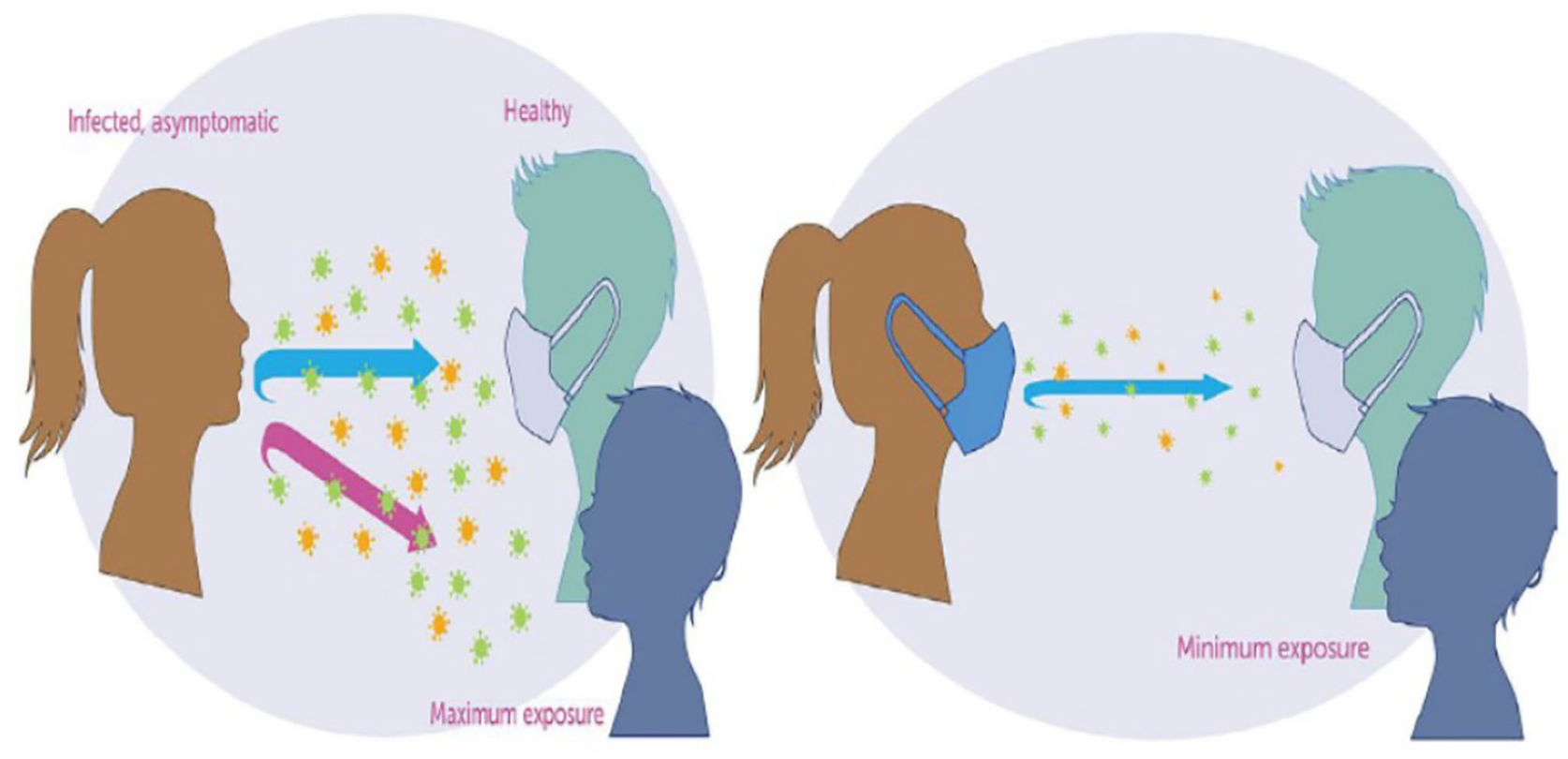

FIGURE 1 | The science of mask against COVID-19 (20).

state of emergency and issuing a state-wide shelter in place. The use of a face mask by the general public in the United States has been controversial as some state governors issued executive orders that voided face mask mandates within their jurisdiction (17).

Numerous mathematical models have been used to provide insights into public health measures for mitigating the spread of the novel coronavirus pandemic. Ferguson et al. (18) proposed an agent-based model to assess the impact of NPIs on COVID19 mortality. In the absence of public health interventions, their model projected high mortality in the United States and the United Kingdom. Eikenberry et al. (3) developed a mathematical model to assess the impact of mask use by the general public on the transmission dynamics of the COVID-19 pandemic. Their results showed that broad adoption of even relatively ineffective face masks might reduce community transmission of COVID19 and decrease peak hospitalizations and deaths. Recently, Ngonghala et al. (8) developed a mathematical model to assess the impact of NPIs on curtailing the public health burden of COVID-19 in the United States. Their study showed the effect of early implementation of face masks, lockdown, and lifting of social-distancing. Extending the duration of lockdown could reduce the daily cases, daily mortality in the United States. Mizumoto and Chowell (19) used a mathematical model to assess the potential for a coronavirus outbreak aboard the Diamond Princess cruise, which experienced a major COVID-19 outbreak during the months of January and February of 2020. Their study showed that the basic reproduction number of the model decreases with increasing the effectiveness of the quarantine and isolation measures implemented on the ship.

Despite public health campaigns regarding the use of a face masks and social-distancing in the United States, the local transmission of COVID-19 throughout different parts of the country continues to rise. While many people follow public health recommendations to the use of face mask and practice social-distance in public to limit the spread of the virus, others passionately fight against them. It is important to understand how educating the population on the importance of using a face mask and social-distancing could reduce the spread of the virus. The objective of this study is to use a mathematical model to assess the impact of public health education campaigns on the coronavirus outbreak in the United States.

\section{MATERIALS AND METHODS}

\section{Model Formulation}

The coronavirus model to be developed uses the natural history of the infection. The total human population at time $t$, denoted by $N(t)$, is sub-divided into mutually exclusive compartments of unwilling susceptible $\left[S_{u}(t)\right]$, willing susceptible $\left[S_{e}(t)\right]$, unwilling exposed $\left[E_{\mathcal{u}}(t)\right]$, willing exposed $\left[E_{e}(t)\right]$, unwilling asymptomatic-infectious $\left[A_{u}(t)\right]$, willing asymptomaticinfectious $\left[A_{e}(t)\right]$, unwilling infectious with symptoms $\left[I_{u s}(t)\right]$, willing infectious with symptoms $\left[I_{e s}(t)\right]$, unwilling hospitalized or isolated at a health care facility $\left[H_{u}(t)\right]$, willing hospitalized or isolated at a health care facility $\left[H_{e}(t)\right]$, in intensive care units $\left[I_{c u}(t)\right]$, and recovered $[R(t)]$ individuals. Thus, the total population size $N$ is given as

$$
\begin{aligned}
N(t)= & S_{u}(t)+S_{e}(t)+E_{u}(t)+E_{e}(t)+I_{u s}(t)+I_{e s}(t)+A_{u}(t) \\
& +A_{e}(t)+H_{u}(t)+H_{e}(t)+I_{c u}(t)+R(t) .
\end{aligned}
$$




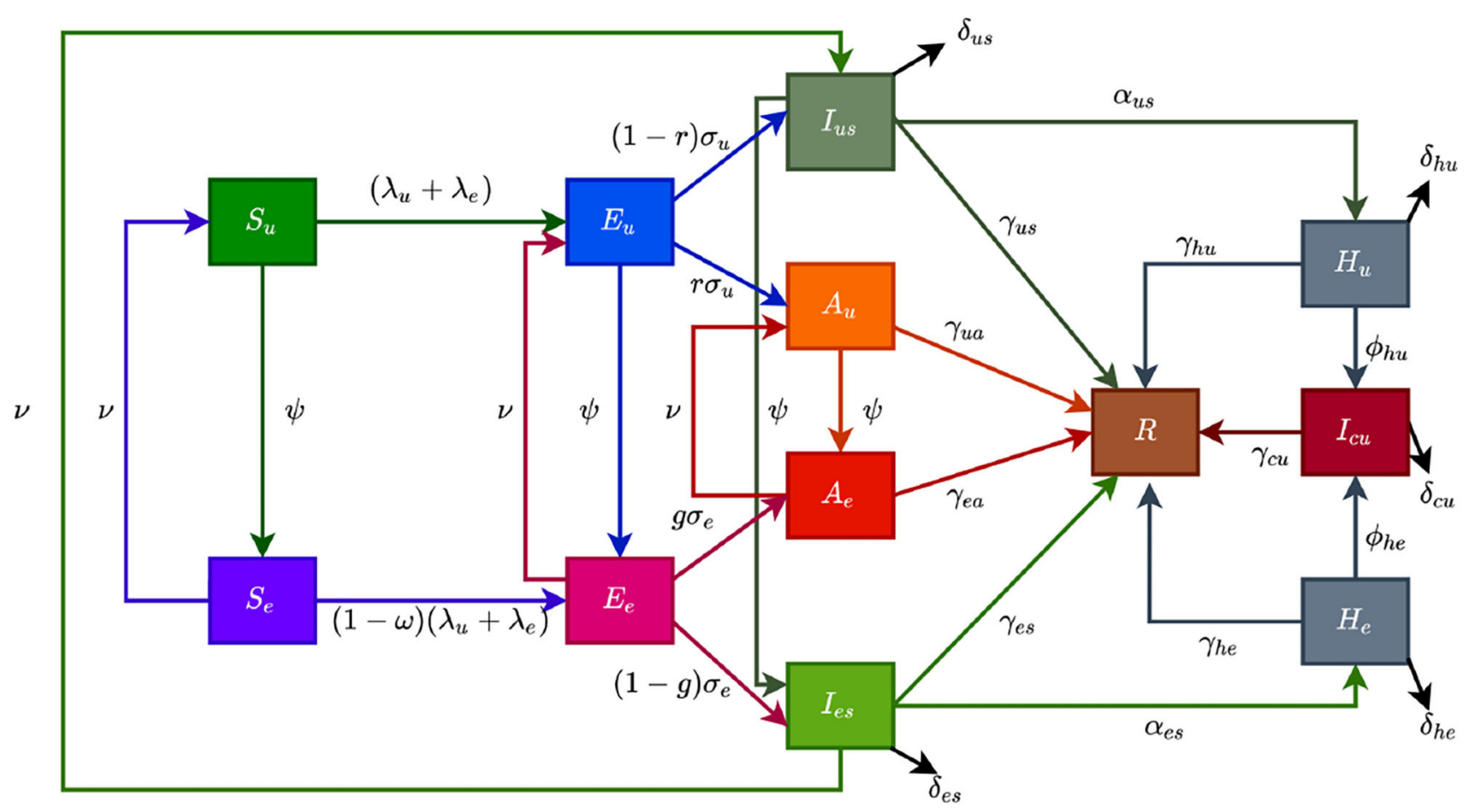

FIGURE 2 | Flow diagram of the model showing transitions from various compartments based on public health education.

TABLE 1 | Description of the state variables of the model (S1).

\begin{tabular}{|c|c|}
\hline $\begin{array}{l}\text { State } \\
\text { variable }\end{array}$ & Description \\
\hline$S_{u}$ & Population of unwilling susceptible individuals \\
\hline$S_{e}$ & Population of willing susceptible individuals \\
\hline$E_{u}$ & Population of unwilling exposed individuals \\
\hline$E_{e}$ & Population of willing exposed individuals \\
\hline Ius & $\begin{array}{l}\text { Population of unwilling infectious individuals with severe clinical } \\
\text { symptoms of COVID-19 }\end{array}$ \\
\hline les & $\begin{array}{l}\text { Population of willing infectious individuals with severe clinical } \\
\text { symptoms of COVID-19 }\end{array}$ \\
\hline$A_{u}$ & Population of unwilling asymptomatic-Infectious individuals \\
\hline$A_{e}$ & Population of willing asymptomatic-Infectious individuals \\
\hline$H_{u}$ & Population of unwilling hospitalized individuals \\
\hline$H_{e}$ & Population of willing hospitalized individuals \\
\hline$I_{c u}$ & Population of individuals in ICU \\
\hline$R$ & Population of recovered individuals \\
\hline
\end{tabular}

The flow diagram of the model (S1) is depicted in Figure 2 (the state variables and parameters of the model are described in Tables 1, 2, respectively).

In model ( $\mathrm{S} 1), \beta$ is the effective infection rate for unwilling and willing individuals, while $\eta_{j},\left(j \in\left\{A_{k}, H_{k}\right\}, k \in\{u, e\}\right)$, is the modification parameters (where $0<\eta_{j}<1$ ) that accounts for a reduction in infectiousness of unwilling(willing) asymptomatic and hospitalized individuals compared to unwilling(willing)
TABLE 2 | Description of parameters of the model (S1).

\begin{tabular}{|c|c|}
\hline Parameter & Description \\
\hline$\beta$ & $\begin{array}{l}\text { Effective contact rates for willing(unwilling) } \\
\text { individuals }\end{array}$ \\
\hline$\omega$ & $\begin{array}{l}\text { Efficacy of education in preventing } \\
\text { COVID-19 infection }(0<\omega \leq 1)\end{array}$ \\
\hline$\eta_{A_{u}}\left(\eta_{A_{e}}\right)\left(\eta_{H_{u}}\right)\left(\eta_{H_{e}}\right)$ & $\begin{array}{l}\text { Modification parameters } \\
\left(0<\eta_{A_{u}}\left(\eta_{A_{e}}\right)\left(\eta_{H_{u}}\right)\left(\eta_{H_{e}}\right)<1\right)\end{array}$ \\
\hline$\psi$ & $\begin{array}{l}\text { Education rate for individuals in } S_{u}\left(E_{u}\right)\left(I_{u s}\right) \\
\left(A_{u}\right)\end{array}$ \\
\hline$v$ & $\begin{array}{l}\text { Fatigue rate (loss of willingness to public } \\
\text { health measures) }\end{array}$ \\
\hline$\sigma_{u}\left(\sigma_{e}\right)$ & $\begin{array}{l}\text { Progression rates from } E_{u}\left(E_{e}\right) \text { to } I_{\text {us }}\left(I_{e s}\right) \text { or } \\
A_{e}\left(A_{e}\right) \text { class }\end{array}$ \\
\hline$r(g)$ & $\begin{array}{l}\text { Proportion of individuals in } E_{u}\left(E_{e}\right) \text { class who } \\
\text { show clinical symptoms of COVID-19 }\end{array}$ \\
\hline$\alpha_{u s}\left(\alpha_{e s}\right)$ & $\begin{array}{l}\text { Hospitalization rates for unwilling(willing) } \\
\text { infectious individuals }\end{array}$ \\
\hline$\phi_{\text {hu }}\left(\phi_{\text {he }}\right)$ & $\begin{array}{l}\text { ICU admission rate for unwilling(willing) } \\
\text { hospitalized individuals }\end{array}$ \\
\hline$\gamma_{u a}\left(\gamma_{e a}\right)\left(\gamma_{u s}\right)\left(\gamma_{e s}\right)\left(\gamma_{h}\right)\left(\gamma_{c u}\right)$ & $\begin{array}{l}\text { Recovery rates for individuals in the } \\
A\left(I_{S}\right)(H)\left(I_{C u}\right) \text { class }\end{array}$ \\
\hline$\delta_{u s}\left(\delta_{e s}\right)\left(\delta_{h}\right)\left(\delta_{c u}\right)$ & $\begin{array}{l}\text { Disease-induced death rates for individuals } \\
\text { in the } I_{\text {us }}\left(I_{\text {es }}\right)(H)\left(I_{c u}\right) \text { class }\end{array}$ \\
\hline
\end{tabular}

symptomatic individuals. Further, $\psi$ represent the public health education rate for unwilling susceptible $\left(S_{u}\right)$, exposed $\left(E_{u}\right)$, symptomatic $\left(I_{u s}\right)$, and asymptomatic individuals $\left(A_{u}\right)$, 
respectively. It is assumed that public health education program toward the use of NPIs in preventing COVID-19 infection is imperfect (i.e., allowing willing susceptible individuals become infected with COVID-19), with an efficacy $\omega$ (where $0<\omega \leq$ 1). Furthermore, the parameters $\sigma_{j}, j=u, e$ represents the progression rates of unwilling (willing) exposed individuals. A proportion, $0<r, g \leq 1$, of unwilling (willing) exposed individuals show clinical symptoms of COVID-19 and move to the class $I_{j s}, j=u, e$, at the end of the incubation period. The remaining proportion, $(1-r)$ and $(1-g)$, show no clinical symptoms and move to the $A_{j}, j=u, e$, class. Further, $v$ represent the loss of willingness to wear a face mask, practice social-distancing in public, and frequently washing hands. The parameters $\alpha_{j s}, j=u, e$, is the hospitalization (or self-isolation) rates of unwilling(willing) individuals with clinical symptoms of COVID-19. Similarly, the parameters $\phi_{h u}, \phi_{h e}$ is the ICU admission rates. The parameters $\gamma_{j a}, \gamma_{j s}, \gamma_{h j}, \gamma_{c u}, j=u, e$, represents the recovery rates for unwilling (willing) individuals in the $A_{j}, I_{j s}, H_{j}, I_{c u}, j=u, e$ classes. Finally, the parameter $\delta_{j s}, \delta_{h j}, \delta_{c u}, j=u, e$ represents the COVID-induced mortality rate for individuals in the $I_{j s}, H_{j}, I_{c u}, j=u, e$ classes. To formulate the model, we made the following assumptions:

(i) due to public health education, willing individuals wear face mask to prevent transmission, practise social-distancing and wash their hands while unwilling individuals do not.

(ii) public health education program is targeted at individuals who are unwilling to use a face mask or practice social-distance in public at rate $(\psi)$.

(iii) to account for public health education saturation, we assume a willingness fatigue (i.e., loss of willingness to wear face mask, practise social-distancing, and frequent washing of hands),

The model (S1) is also an extension of the COVID-19 models in $(3,5,8-10)$ by including compartments for individuals based on their willingness/unwillingness regarding the adherence to non-pharmaceutical interventions such as face mask, socialdistancing, and hand washing to curtail the COVID-19 outbreak. Models of this type have been formulated for Influenza (12) and COVID-19.

\section{RESULTS}

\section{Asymptotic Stability of Disease-Free Equilibria}

The expression for the reproduction number $\left(\mathcal{R}_{c}\right)$ for model with public health education program is given in the Supplementary Material.

Theorem 0.1. The disease-free equilibrium (DFE) of the model (S1) is locally-asymptotically stable if $\mathcal{R}_{c}<1$. If $\mathcal{R}_{c}>1$, the epidemic grows rapidly, reaches a peak, and eventually declines to zero.

The quantity $\mathcal{R}_{c}$ is the reproduction number of the model (S1). It measures the average number of new COVID-19 cases generated by a typical infectious individual introduced into a population where a certain fraction is protected.

\section{Data Fitting and Parameter Estimation}

Estimates for some of the parameters of the model (S1) were obtained from the literature (as indicated in Table 3). Other parameters, such as the effective infection rate parameters $\beta$, education rate $\psi$, education efficacy $\omega$, and fatigue rate $v$ are obtained by fitting the model to the observed cumulative mortality data for the United States $(21,22)$. In particular, the United States Cumulative mortality data from January 22, 2020 (first index case) to December 8, 2020 were obtained from the John Hopkins Center for Systems Science and Engineering COVID-19 Dashboard (23). We fitted the model for three different time periods of the pandemic, with the first period from January 22, 2020 to July 5, 2020, second period from July 6, 2020 to September 30, 2020, and the third period from October 1, 2020 to December 8,2020 . This was done in order to correctly capture the trends observed in the daily mortality data (i.e., the COVID19 waves observed). Hence, we obtained three set of values for the parameters to be estimated based on the different periods. Our choice of fitting the model to the mortality data is due to the fact that there is evidence of under-reporting and undertesting of COVID-19 cases in countries such as France, Italy, United States, Iran, and Spain. Hence, mortality data may provide a better indicator for COVID-19 case spread $(8,24)$. The datafitting process involves implementing the standard nonlinear least squares approach using the fmincon Optimization Toolbox embedded in MATLAB. The estimated values of the unknown parameters are tabulated in Table 4. Figures 3A-C depicts the fitting of the observed and predicted cumulative mortality for the United States. Further, Figures 3D,E compares the simulations of the model using the fitted and fixed parameter in Tables 3 , 4. The results depicted in Figure 3, show that the model also captures the observed daily mortality data for each of the period considered. Thus, the parameter estimation of model (S1) shows that cumulative mortality data provides a very reliable calibration for coronavirus transmission dynamics. In Figures $\mathbf{3 A , D}$, it is worth mentioning that the fit is not really good around mid March. This is not surprising since testing capacities have been ramped up around this time, leading to an increasing fraction of infections being detected.

\section{Sensitivity Analysis}

The model (S1) contains parameters, and uncertainty in their estimates are expected to arise. The effect of such uncertainties is assessed using uncertainty and sensitivity analysis (25-27). In particular Latin Hypercube Sampling (LHS) and Partial Rank Correlation Coefficients (PRCC) is used to identify model parameters that have the most influence on the model with the reproduction number $\left(\mathcal{R}_{c}\right)$ as the response function. The purpose of this analysis is to determine effects of parameters on model outcomes (25-27). A highly sensitive parameter should be more carefully estimated, since a small change in that parameter can cause a large quantitative changes in the result (25-27). On the other hand, a parameter that is not sensitive does not require as much attempt to estimate, since a small change in that parameter will not cause a large variation to the quantity of interest (26). Parameters with large PRCC greater than +0.50 are said to be highly positively correlated with the response 
function, while those $<-0.50$ are said to be highly negatively correlated with the response function (25-27). The parameters considered in the PRCCs analysis are the effective infection rate for unwilling (willing) individuals $(\beta)$, education rates for unwilling (willing) individuals $(\psi)$, education efficacy $(\omega)$, and fatigue rate $(v)$. We performed a PRCC analysis for the three different periods; however, the parameters have the same effect on the response function for the three periods. We chose to report one plot as displayed in Figure 4. The results show that the

TABLE 3 | Baseline parameter values for the model (S1) drawn from the literature.

\begin{tabular}{lcc}
\hline Fixed Parameter $(\boldsymbol{k}=\boldsymbol{u}, \boldsymbol{e})$ & Value & References \\
\hline$\sigma_{e}, \sigma_{u}$ & $1 / 2.5 /$ day & $(31,32)$ \\
$r, g$ & 0.35 & $(33,34)$ \\
$\eta_{A_{k}}$ & 1.5 & Assumed \\
$\eta_{H_{k}}$ & 0.25 & Assumed \\
$\alpha_{u s}, \alpha_{e s}$ & $1 / 6 /$ day & $(35)$ \\
$\phi_{h u}, \phi_{\text {he }}$ & $0.083 /$ day & $(36)$ \\
$\gamma_{u a}, \gamma_{e a}$ & $1 / 5 /$ day & $(35)$ \\
$\gamma_{u s}, \gamma_{e s}$ & $1 / 10 /$ day & $(18,37)$ \\
$\gamma_{\text {hu }}, \gamma_{\text {he }}$ & $1 / 8 /$ day & $(18)$ \\
$\gamma_{c u}$ & $1 / 10 /$ day & $(18,37)$ \\
$\delta_{k s}$ & $0.015 /$ day & $(3,5,18)$ \\
$\delta_{h k}$ & $0.015 /$ day & $(3,5,18)$ \\
$\delta_{C u}$ & $0.0225 /$ day & $(3,5,18)$
\end{tabular}

four parameters that mostly impact the response function $\left(\mathcal{R}_{c}\right)$ are the effective infection rate $(\beta)$, education rate $(\psi)$, fatigue rate $(\nu)$, and education efficacy $(\omega)$. Based on the PRCC values, the transmission rate for unwilling individuals and the fatigue rate has a positive impact on $\mathcal{R}_{c}$, as an increase(decrease) in the transmission and fatigue parameter will increase(decrease) $\mathcal{R}_{c}$. In contrast, the education rate and efficacy have a negative impact on the $\mathcal{R}_{c}$, and an increase in these parameters will decrease the $\mathcal{R}_{c}$.

\section{Numerical Simulation Results}

To capture the trends observed in the daily mortality data obtained for the United States from January 22, 2020, to December 8, 2020, we considered three different periods of the pandemic with the first period from January 22, 2020, to July 5, 2020, second period from July 6, 2020 to September 30, 2020, and the third period from October 1, 2020 to December 8, 2020. First,

TABLE 4 | Estimated parameter values for the model (S1) using COVID-19 mortality data for the United States.

\begin{tabular}{lccc}
$\begin{array}{l}\text { Estimated } \\
\text { Parameters }\end{array}$ & $\mathbf{1 / 2 2 / 2 0 2 0 - 7 / 5 / 2 0 2 0}$ & $\mathbf{7 / 6 / 2 0 2 0 - 9 / 3 0 / 2 0 2 0 ~}$ & $\mathbf{1 0 / 1 / 2 0 2 0 - 1 2 / 8 / 2 0 2 0}$ \\
\hline$\beta$ & 0.8084 & 0.4369 & 0.2842 \\
$\psi$ & 0.0279 & 0.0781 & 0.0249 \\
$\nu$ & 0.0011 & 0.0210 & 0.0461 \\
$\omega$ & 0.8982 & 0.8599 & 0.8896
\end{tabular}

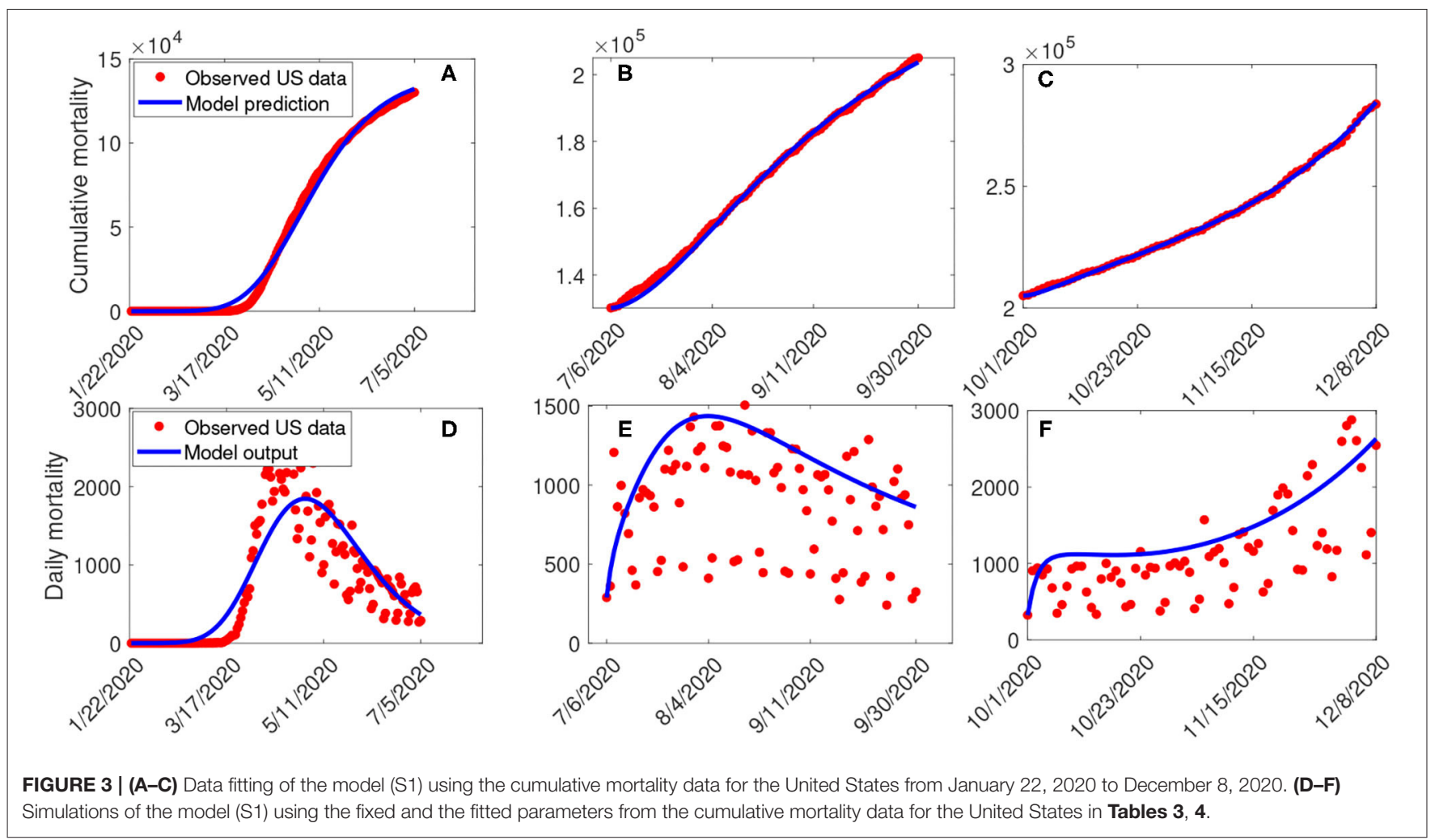




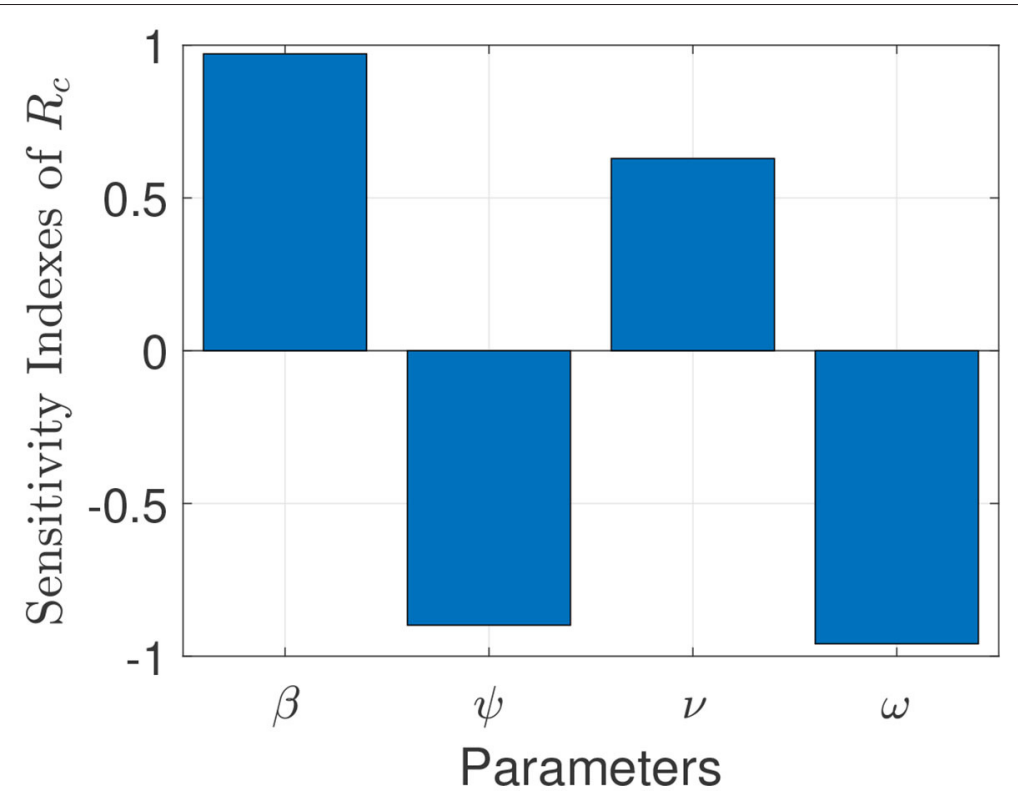

FIGURE 4 | Partial rank correlation coefficients (PRCCs) showing the impact of four model parameters on the reproduction number ( $\mathcal{R}_{C}$ ) of the model. Parameter values used are as given in Tables 3, 4 .

we generated a contour plot of the reproduction number $\left(\mathcal{R}_{c}\right)$ of the model (S1), as a function of education rate $(\psi)$ and education efficacy $(\omega)$ (Figure 5). Figure 5a for the period January 22, 2020 to July 5, 2020, suggests that the control reproduction number $\mathcal{R}_{c}$ is practically independent of $\psi$ ( at least for $\psi \ll 1$ ). A similar trend is observed for the period July 6, 2020, to September 30, 2020, of the outbreak (Figure 5b). However, Figure 5c shows that for the period October 1, 2020, to December 8, 2020, as more people are being educated with high efficacy, the value of $\mathcal{R}_{c}$ decreases. It is worth mentioning that the value of $\mathcal{R}_{c}$ depends on the initial conditions, more precisely on the location of the specific DFE within the hyperplane of disease-free equilibria. Assuming that no individuals are educated at the beginning of the simulation, then the education efficacy $(\omega)$ will be irrelevant (sensitivity index close to zero) since in the beginning there are no individual that have already been educated. This impedes the immediate curtailment of the epidemic much more severely than too few individuals being in the process of being educated (this is exactly the reason, why $\psi$ does barely affect $\mathcal{R}_{c}$ : rather than the flux from uneducated to educated individuals the number of currently educated individuals acts on $\mathcal{R}_{c}$ ). As soon as a significant number of individuals is educated, the effect of the education efficacy on $\mathcal{R}_{c}$ will increase dramatically. Moreover, since $\mathcal{R}_{c}$ depends on the values of the initial conditions ( $S_{u}^{*}$ and $S_{e}^{*}$ ), it is expected that $\mathcal{R}_{c}$ decreases as more individuals are being educated over time.

Figure 6 depicts a contour plot of the reproduction number $\left(\mathcal{R}_{c}\right)$ of the model (S1), as a function of the proportion of educated individuals among all susceptible $\left(\frac{S_{e}^{*}}{S_{u}^{*}+S_{e}^{*}}\right)$ and education efficacy $(\omega)$ for a fixed education rate $(\psi)$. Figure 6a shows that for the period January 22, 2020 to July 5, 2020, with the baseline education efficacy, $\mathcal{R}_{c}$ can be brought to a value $<1$ if $90 \%$ among all susceptible individuals are educated. This result suggests that an incredibly high education rate $(\psi)$ is necessary to curtail the outbreak effectively for the period January 22, 2020 , to July 5, 2020. However, for the period July 6, 2020, to September 30, 2020, of the outbreak, with the baseline education efficacy, $\mathcal{R}_{c}$ can be brought to a value less than one if $76 \%$ among all susceptible individuals are educated (Figure $\mathbf{6 b}$ ). Figure $\mathbf{6 c}$ shows that for the period October 1, 2020, to December 8, 2020, with the baseline education efficacy, $\mathcal{R}_{c}$ can be brought to a value less than one if $51 \%$ among all susceptible individuals are educated. This result further supports the need to educate more people if we are to effectively curtail the coronavirus outbreak, which is consistent with the results obtained in Figure 5.

Furthermore, we ran simulations of model (S1) using the parameter values in Tables 4, 5, to assess the population-level impact of public health education program on the COVID19 outbreak. The simulation result for the baseline scenario shows a projected 132,000 cumulative deaths by July 5, 2020, 205,600 by September 30, 2020, and 285,100 by December 8 , 2020 (Figure 7A). Similarly, the projected peak daily mortality was 1,829 attained on April 28, 2020, 1,505 attained on August 3, 2020, and 2,808 attained by December 8, 2020 (Figure 7B). Further, with a $10 \%$ increase in education rate from the baseline value, Figure 7A shows a projected 44,400 cumulative mortality by July $5,2020,105,300$ by September 30,2020 , and 178,000 by December 8,2020 . This result is approximately a $66.4 \%$ reduction in cumulative mortality by July 5,2020 , a $48.8 \%$ reduction in cumulative mortality by September 30,2020 , a $37.6 \%$ reduction in cumulative mortality by December 8,2020 , when compared to the baseline scenario. Figure $7 \mathbf{B}$ with a $10 \%$ increase in education 

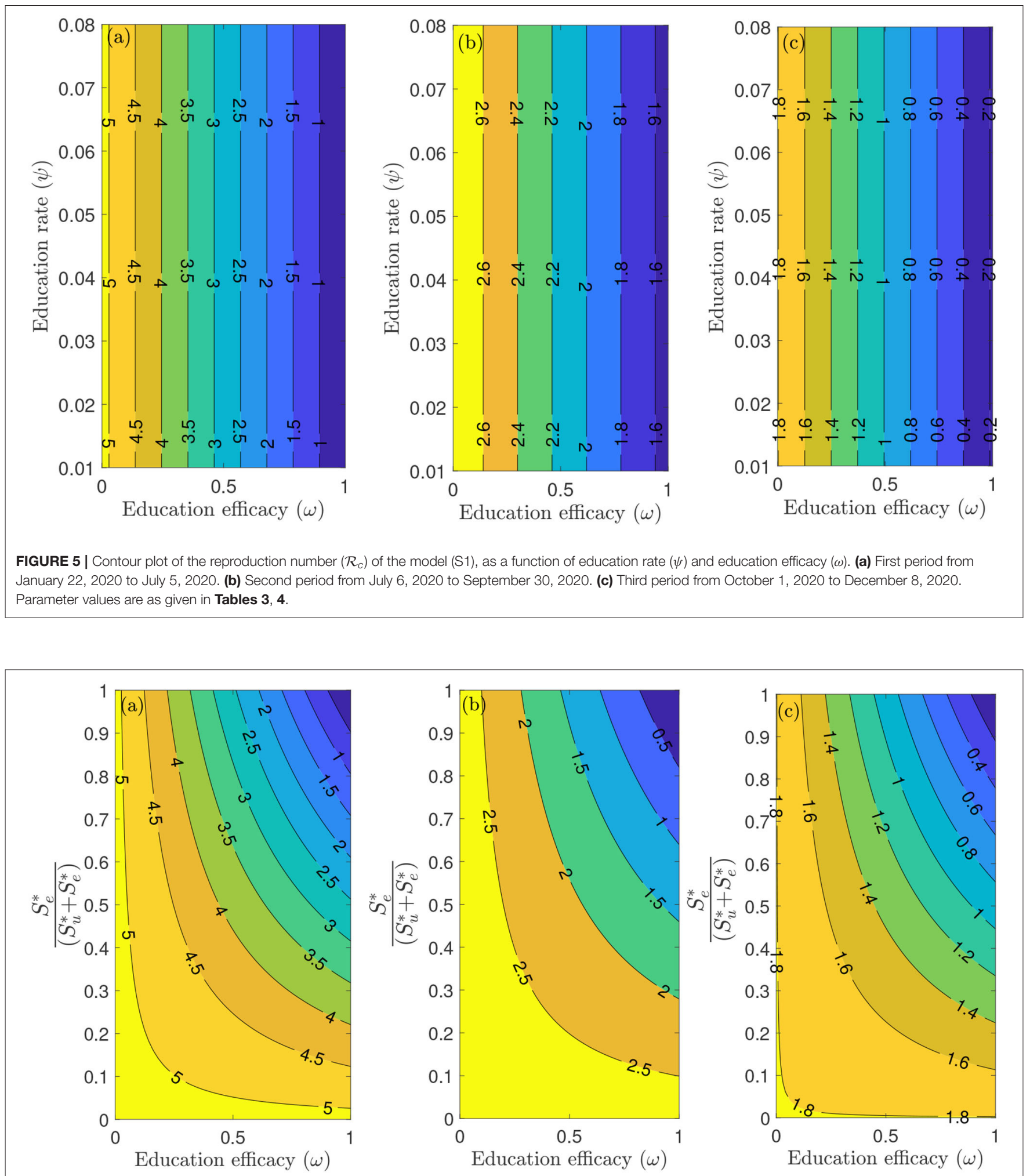

FIGURE 6 | Contour plot of the reproduction number $\left(\mathcal{R}_{c}\right)$ of the model $(S 1)$, as a function of different ratios of $\left(\frac{S_{*}^{*}}{\left(S_{*}^{*}+S_{*}^{*}\right)}\right)$ and education efficacy $(\omega)$ with a fixed education rate $\psi$. (a) First period from January 22, 2020 to July 5, 2020. (b) Second period from July 6, 2020 to September 30, 2020. (c) Third period from October 1, 2020 to December 8, 2020. Parameter values are as given in Tables 3, 4. 
TABLE 5 | A summary of various increase in education rate.

\begin{tabular}{|c|c|c|c|c|c|c|}
\hline \multirow[b]{2}{*}{ Education rate } & \multicolumn{2}{|c|}{$1 / 22 / 2020-7 / 5 / 2020$} & \multicolumn{2}{|c|}{$7 / 6 / 2020-8 / 30 / 2020$} & \multicolumn{2}{|c|}{$10 / 1 / 2020-12 / 8 / 2020$} \\
\hline & cum. mort. & daily mort. & cum. mort. & daily mort. & cum. mort. & daily mort. \\
\hline Baseline & 132,000 & 1,829 & 205,600 & 1,505 & 285,100 & 2,808 \\
\hline $10 \%$ increase in $\psi$ & 44,400 & 617 & 105,300 & 1,103 & 178,000 & 2,359 \\
\hline $20 \%$ increase in $\psi$ & 17,210 & 248 & 69,160 & 962 & 136,200 & 1,867 \\
\hline $30 \%$ increase in $\psi$ & 7,676 & 115 & 53,060 & 887 & 115,200 & 1,526 \\
\hline $40 \%$ increase in $\psi$ & 3,835 & 58 & 44,410 & 836 & 102,200 & 1,268 \\
\hline
\end{tabular}

rate from the baseline value, shows projected 617 peak mortality by April 20, 2020, 1,103 by July 31, 2020, and 2,359 by December 8,2020 . This result is approximately a $66.3 \%$ reduction in peak daily mortality by April, 20, 2020, a 26.7\% reduction in peak daily mortality by July 31,2020 , a $16 \%$ reduction in peak daily mortality by December 8, 2020 when compared to the baseline scenario. However, with a $40 \%$ increase in education rate from the baseline value, Figure 7A shows a projected 3,835 cumulative mortality by July $5,2020,44,410$ by September 30,2020 , and 102,200 by December 8,2020 . This result is approximately a $97.1 \%$ reduction in cumulative mortality by July 5,2020 , a $78.4 \%$ reduction in cumulative mortality by September 30, 2020, a $64.2 \%$ reduction in cumulative mortality by December 8,2020 , when compared to the baseline scenario. Figure 7 B with a $40 \%$ increase in education rate from the baseline value, shows projected 58 peak mortality by April 7, 2020, 836 by July 24, 2020, and 1,268 by December 8,2020 . This result is approximately a $96.8 \%$ reduction in peak daily mortality by April, 7,2020 , a $44.5 \%$ reduction in peak daily mortality by July 24,2020 , a $54.8 \%$ reduction in peak daily mortality by December 8,2020 , when compared to the baseline scenario. The result in Figure 7 shows the need for an aggressive public health education program toward the use of NPIs to curtail the spread of the virus. A summary of the impact of various increase in education rate on cumulative mortality and peak daily mortality is tabulated in Table 5 .

Figure 8 depicts the impact of the loss of willingness to public health measures on COVID-19 outbreak. The result shows that with a $10 \%$ increase in fatigue rate from the baseline value, Figure 8A projected 144,100 cumulative mortality by July $5,2020,228,800$ by September 30,2020 , and 325,700 by December 8,2020 . This result is approximately a $9.2 \%$ increase in cumulative deaths by July 5, 2020, a $11.3 \%$ increase in cumulative deaths by September 30,2020, and a $14.2 \%$ increase in cumulative deaths by December 8, 2020, when compared to the baseline scenario. Figure 8B with a $10 \%$ increase in fatigue rate from the baseline value, shows projected 1,955 peak mortality by May 1, 2020, 1,657 by August 3, 2020, and 4,451 by December 8, 2020. This result is approximately a $6.9 \%$ increase in peak daily mortality by May 1, 2020, a $10.1 \%$ increase in peak daily mortality by August 3, 2020, a 58.5\% increase in peak daily mortality by December 8,2020 , when compared to the baseline scenario. However, with a $40 \%$ increase in fatigue rate from the baseline value, Figure 8A shows a projected 184,200 cumulative mortality by July $5,2020,313,200$ by September 30,2020 , and 478,300 by December 8,2020 . This result is approximately a $39.5 \%$ increase in cumulative deaths by July 5,2020 , a $52.3 \%$ increase in cumulative deaths by September 30,2020, and a $67.8 \%$ increase in cumulative deaths by December 8, 2020 when compared to the baseline scenario. Figure 8B with a $40 \%$ increase in fatigue rate from the baseline value, shows projected 2,412 peak mortality by May 3, 2020, 2,513 by August 23, 2020, and 9,935 by December 8,2020 . This result is approximately a $31.9 \%$ increase in peak daily mortality by April, 20, 2020, a 67\% increase in peak daily mortality by July 27,2020 , a $254 \%$ increase in peak daily mortality by December 8,2020 , when compared to the baseline scenario. This result suggests the need to obey public health measures as loss of willingness would increase the cumulative and daily mortality in the United States. A summary of the impact of the various increase in fatigue rate on cumulative mortality and peak daily mortality is tabulated in Table 6.

\section{DISCUSSION AND CONCLUSIONS}

In this study, we developed a mathematical model for the transmission dynamics and control of COVID-19 in the United States by stratifying the total population into two subgroups of willing and unwilling individuals to the use of face masks, social-distancing in public, and proper/frequent hand washing. The model allows for the assessment of the impact of public health education programs on the coronavirus outbreak in the United States. The model was parameterized using cumulative mortality data for the United States from January 22, 2020, to December 8, 2020, to assess the population-level impact of public health education programs on the outbreak. In particular, we showed that the disease-free equilibrium of the model is locally-asymptotically stable whenever a certain epidemiological threshold, known as the reproduction number $\left(\mathcal{R}_{c}\right)$ is less than one. The epidemiological implication of this result is that when $\mathcal{R}_{c}<1$, a small COVID-infected individuals in the community will not lead to an outbreak.

We explored the sensitivity of the reproduction number with respect to public health education rate in the United States for three different periods of the outbreak. In particular, we showed that community transmission of COVID-19 could be significantly reduced with a very high education rate. In other words, our study shows that COVID-19 could have been effectively controlled if the public health education campaign has been intensified enough with high efficacy (and sustained) from the beginning of the pandemic. Furthermore, we also explored the sensitivity of the reproduction number with respect 

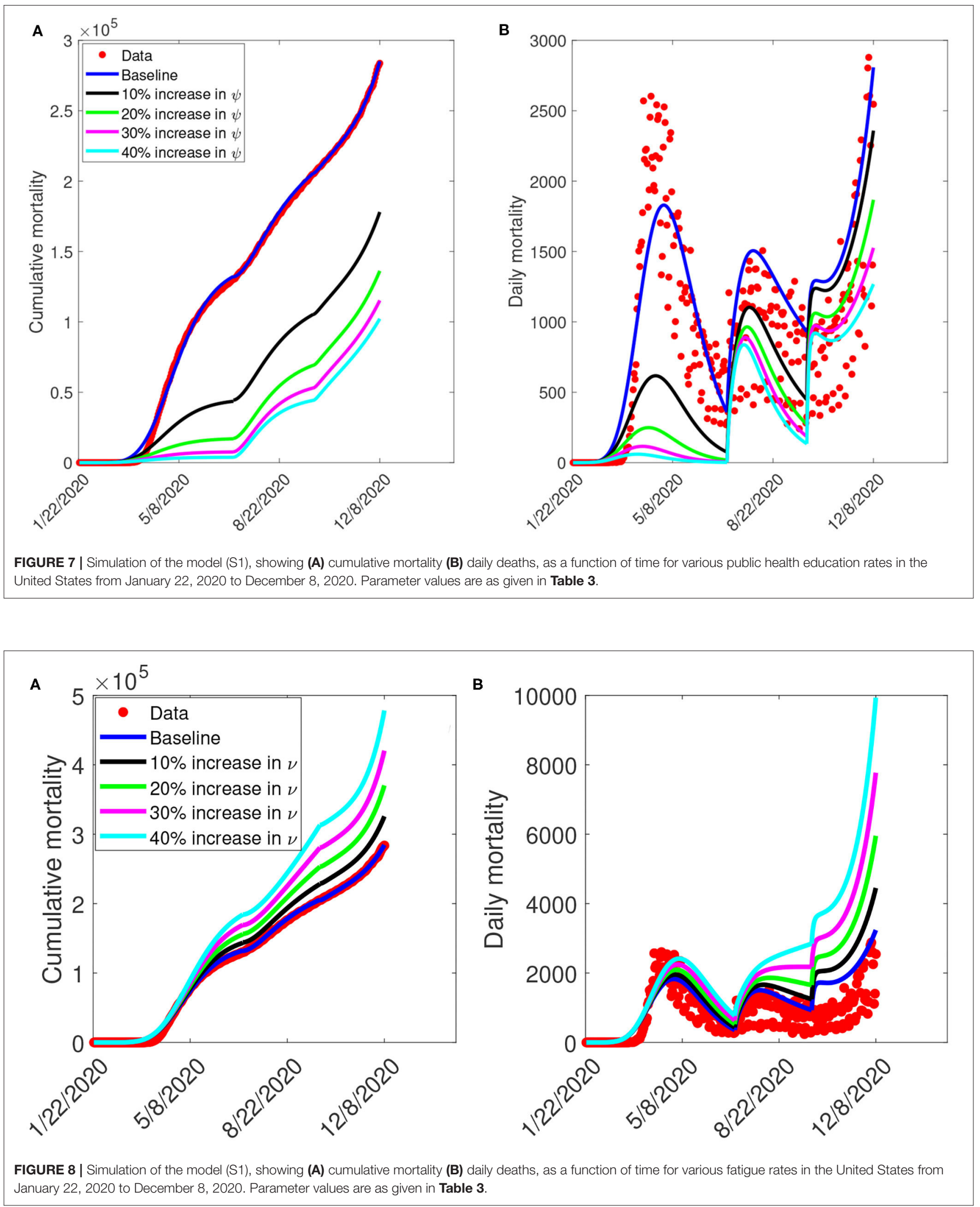
TABLE 6 | A summary of various increase in fatigue rate.

\begin{tabular}{|c|c|c|c|c|c|c|}
\hline \multirow[b]{2}{*}{ Fatigue rate } & \multicolumn{2}{|c|}{$1 / 22 / 2020-7 / 5 / 2020$} & \multicolumn{2}{|c|}{$7 / 6 / 2020-8 / 30 / 2020$} & \multicolumn{2}{|c|}{$10 / 1 / 2020-12 / 8 / 2020$} \\
\hline & cum. mort. & daily mort. & cum. mort. & daily mort. & cum. mort. & daily mort. \\
\hline Baseline & 132,000 & 1,829 & 205,600 & 1,505 & 285,100 & 2,808 \\
\hline $10 \%$ increase in $v$ & 144,100 & 1,955 & 228,800 & 1,657 & 325,700 & 4,451 \\
\hline $20 \%$ increase in $v$ & 157,400 & 2,094 & 253,200 & 1,860 & 370,300 & 5,953 \\
\hline $30 \%$ increase in $v$ & 169,100 & 2,247 & 280,600 & 2,139 & 420,400 & 7,771 \\
\hline $40 \%$ increase in $v$ & 184,200 & 2,412 & 313,200 & 2,513 & 478,300 & 9,935 \\
\hline
\end{tabular}

to willingness fatigue rate in the United States for three different periods of the outbreak. Since the reproduction number $\mathcal{R}_{c}$ depends on the values of the initial conditions $\left(S_{u}^{*}\right.$ and $\left.S_{e}^{*}\right)$, our result shows that $\mathcal{R}_{c}$ can be brought to a value less than one (needed to effectively control the disease) as more individuals are being educated over time.

We also assessed the impact of public health education on the outbreak. Our simulation shows that the possibility of curtailing the spread of the virus (bringing $\mathcal{R}_{c}<1$ ) in the United States is dependent on a very high education rate with high efficacy. The results obtained further showed the prospect of effective public health education programs in reducing both the cumulative and daily mortality of the novel coronavirus in the United States. In particular, a $10 \%$ increase in education rate from the baseline value reduces the peak mortality by $66.3 \%$ by April 20, 2020, $26.7 \%$ by July 31,2020 , and $16 \%$ by December 8,2020 , when compared to the baseline scenario. However, a $40 \%$ increase in education rate from the baseline value reduces the peak daily mortality by $96.8 \%$ by April 7, 2020, 44.5\% by July 24, 2020, and $54.8 \%$ by December 8,2020 . This result is consistent with what was obtained in $(3,5,8,18)$, where the universal use of face masks greatly curtailed community transmission of COVID-19 and brought the pandemic under very effective control.

The Centers for Disease Control and Prevention (CDC) at the early stage of the pandemic recommended the use of a face mask, social-distancing in public, and proper/frequent hand washing to curtail the spread of the novel coronavirus caused by SARSCoV-2 (3, 6, 28). Many state governments issued executive order mandating a face mask in public and restricting large gatherings of people. However, using a face mask and social-distancing in public places appears to be politicized in the United States (29). In particular, states like Georgia and Iowa barred Mayors and City Councils from introducing mask mandates, even as cases continues to rise in various counties in the state (30). While many people strictly adhere to public health measures, others passionately ignore them. We ran simulations to show the impact of loss of willingness (fatigue rate) on both the cumulative and peak daily mortality. The result indicates that non-compliance to public health measures would increase the cumulative and daily mortality in the United States. In particular, a $10 \%$ increase in fatigue rate from the baseline value increases the peak daily mortality by $6.9 \%$ by May $1,2020,10.1 \%$ by August
3, 2020, and $58.5 \%$ by December 8,2020 , when compared to the baseline scenario. However, a $40 \%$ increase in fatigue rate from the baseline value increases the peak daily mortality by $31.9 \%$ by April 20, 2020, $67 \%$ by July 27, 2020, and $254 \%$ by December 8,2020, when compared to the baseline scenario. This result further supports the fact that states with less adherence to public health measures may experience more coronavirus cases and daily mortality than places where there is strict adherence $(3,5,8)$.

\section{DATA AVAILABILITY STATEMENT}

Publicly available datasets were analyzed in this study. This data can be found at: John Hopkins University; https://github.com/ CSSEGISandData/COVID-19.

\section{AUTHOR CONTRIBUTIONS}

EI, AR, RR, DI, JC, JH, MM, RP, and ZD conceived the study. EI and FA designed the model. EI collected and analyzed the data. EI and BO performed the numerical simulations. EI, AR, RR, DI, JC, JH, MM, RP, ZD, FA, BO, and LA drafted the manuscript. EI, AR, RR, DI, JC, JH, MM, RP, ZD, FA, BO, and LA revised the manuscript. All authors read and approved the final manuscript.

\section{FUNDING}

EI, AR, and RR would like to acknowledge and thank our partners of The Boeing Company and the Thurgood Marshall College Fund, for their charitable, capacity grant in support of the Math RaMP Program at Spelman College, via the Boeing | TMCF HBCU Investment. We would also like to acknowledge the MAA Tensor Women and Mathematics Program for its grant in support of the Math RaMP Program at Spelman College. FA was supported by the National Science Foundation under grant number DMS 2028297.

\section{SUPPLEMENTARY MATERIAL}

The Supplementary Material for this article can be found online at: https://www.frontiersin.org/articles/10.3389/fpubh. 2021.630974/full\#supplementary-material 


\section{REFERENCES}

1. WHO. Novel Coronavirus (2019-nCoV) Situation Reports. WHO. (2020). Available online at: https://www.who.int/emergencies/diseases/novelcoronavirus-2019/situation-reports/

2. WHO. Emergencies, Preparedness, Response. Pneumonia of Unknown Origin96China. Disease Outbreak News. (2020). Available online at: https:// www.who.int/csr/don/05-january-2020-pneumonia-of-unkown-causechina/en/

3. Eikenberry SE, Muncuso M, Iboi E, Phan T, Kostelich E, Kuang Y, et al. To mask or not to mask: modeling the potential for face mask use by the general public to curtail the COVID-19 pandemic. Infect Dis. Model. (2020) 5:293-308. doi: 10.1016/j.idm.2020.04.001

4. WHO. Coronavirus Disease (COVID-19) Dashboard. (2020). Available online at: https://covid19.who.int/region/amro/country/us

5. Ngonghala CN, Iboi E, Eikenberry S, Scotch M, MacIntyre CR, Bonds $\mathrm{MH}$, et al. Mathematical assessment of the impact of non-pharmaceutical interventions on curtailing the 2019 novel Coronavirus. Math Biosci. (2020) 2020:108364. doi: 10.1016/j.mbs.2020.108364

6. Symptoms of Coronavirus (2020). Available online at: https://www.cdc.gov/ coronavirus/2019-ncov/symptoms-testing/symptoms.html

7. John Hopkins University. Daily State-by-State Testing Trends. John Hopkins University Coronavirus Resource Center. (2020). Available online at: https:// coronavirus.jhu.edu/testing/individual-states/usa

8. Ngonghala CN, Iboi EA, Gumel AB. Could masks curtail the post-lockdown resurgence of COVID-19 in the US? Math Biosci. (2020) 329:108452. doi: 10.1016/j.mbs.2020.108452

9. Iboi EA, Ngonghala $\mathrm{CN}$, Gumel $\mathrm{AB}$. Will an imperfect vaccine curtail the COVID-19 pandemic in the US? Infect Dis Model. (2020) 5:510-24. doi: 10.1016/j.idm.2020.07.006

10. Iboi EA, Sharomi OO, Ngonghala CN, Gumel AB. Mathematical modeling and analysis of COVID-19 pandemic in Nigeria. Math Biosci Eng. (2020) 17:7192-220. doi: 10.1101/2020.05.22.20110387

11. Heymann DL, Shindo N. COVID-19: what is next for public health? Lancet. (2020) 395:542-5. doi: 10.1016/S0140-6736(20)30374-3

12. Brauer F, Castillo-Chavez C, Feng Z. Mathematical Models in Epidemiology. New York, NY: Springer (2019). doi: 10.1007/978-1-4939-9828-9

13. Chen W, Wang Q, Li Y, Yu H, Xia Y, Zhang M, et al. Early containment strategies and core measures for prevention and control of novel coronavirus pneumonia in China. Zhonghua yu Fang yi Xue za Zhi. (2020) 54:1-6. doi: 10.3760/cma.j.issn.0253-9624.2020.03.003

14. Gostin L. Public health strategies for pandemic influenza: ethics and the law. JAMA. (2006) 295:1700-4. doi: 10.1001/jama.295.14.1700

15. Group WHOW. Nonpharmaceutical interventions for pandemic influenza, international measures. Emerg Infect Dis. (2006) 12:81. doi: 10.3201/eid1201.051370

16. Group WHOW. Nonpharmaceutical interventions for pandemic influenza, national and community measures. Emerg Infect Dis. (2006) 12:88. doi: 10.3201/eid1201.051371

17. Cohen S. Georgia Unmasked? Gov. Brian Kemp Invalidates Local Mask Orders For 1.4 Million Residents. Forbes. (2020). Available online at: https:// www.forbes.com/sites/sethcohen/2020/07/16/georgia-gov-brian-kempinvalidates-local- mask-orders/?sh $=464 \mathrm{a} 5 \mathrm{a} 0 \mathrm{e} 7 \mathrm{~d} 9 \mathrm{~b}$

18. Ferguson NM, Laydon D, Nedjati-Gilani G, Imai N, Ainslie K, Baguelin M, et al. Impact of Non-Pharmaceutical Interventions (NPIs) to Reduce COVID19 Mortality and Healthcare Demand. London: Imperial College COVID-19 Response Team (2020).

19. Mizumoto K, Chowell G. Transmission potential of the novel coronavirus (COVID-19) onboard the Diamond Princess Cruises Ship, 2020. Infect Dis Model. (2020) 5:264-70. doi: 10.1101/2020.02.24.20027649

20. McCarthy A. How Masks Protect Against COVID-19: Understanding the Science. Boston Children's Hospital (2020). Available online at: https:// discoveries.childrenshospital.org/covid-19-mask-science/

21. Banks HT, Davidian M, Samuels JR, Sutton KL. An inverse problem statistical methodology summary. In: Chowell G, Hyman JM, Bettencourt LMA, Castillo-Chavez C, editors. Mathematical and Statistical
Estimation Approaches in Epidemiology. Dordrecht: Springer (2009). doi: 10.1007/978-90-481-2313-1_11

22. Chowell G. Fitting dynamic models to epidemic outbreaks with quantified uncertainty: a primer for parameter uncertainty, identifiability, and forecasts. Infect Dis Model. (2017) 2:379-98. doi: 10.1016/j.idm.2017.08.001

23. COVID-19 Data Repository by the Center for Systems Science and Engineering (CSSE) at Johns Hopkins University. Github Repository. (2020). Available online at: https://github.com/CSSEGISandData/COVID-19

24. Lau H, Khosrawipour T, Kocbach P, Ichii H, Bania J, Khosrawipour $\mathrm{V}$. Evaluating the massive underreporting and undertesting of COVID19 cases in multiple global epicenters. Pulmonology. (2020) 27:110-5. doi: 10.1016/j.pulmoe.2020.05.015

25. Cariboni J, Gatelli D, Liska R, Saltelli A. The role of sensitivity analysis in ecological modelling. Ecol Model. (2007) 203:167-82. doi: 10.1016/j.ecolmodel.2005.10.045

26. Blower SM, Dowlatabadi H. Sensitivity and uncertainty analysis of complex models of disease transmission: an HIV model, as an example. Int Stat Rev. (1994) 229-43. doi: 10.2307/1403510

27. Iboi EA, Gumel AB. Mathematical assessment of the role of Dengvaxia vaccine on the transmission dynamics of dengue serotypes. Math Biosci. (2018) 304:25-47. doi: 10.1016/j.mbs.2018.07.003

28. Rourke M. CDC Recommends Masks, U.S. Deaths Rise by More Than 1,000 in One Day. (2020). Available online at: https://www.nbcnews.com/health/ health-news/live-blog/2020-04-03-coronavirus-news-n1175641

29. Guardian T. How Did Face Masks Become a Political Issue in America? (2020). Available online at: https://www.theguardian.com/world/2020/jun/29/facemasks-us-politics-coronavirus

30. North A. Why Masks Are (Still) Politicized in America. (2020). Available online at: https://www.vox.com/2020/7/21/21331310/mask-masks-trump-covid19rule-georgia-alabama

31. Linton NM, Kobayashi T, Yang Y, Hayashi K, Akhmetzhanov AR, Jung Sm, et al. Incubation period and other epidemiological characteristics of 2019 novel coronavirus infections with right truncation: a statistical analysis of publicly available case data. J Clin Med. (2020) 9:538. doi: 10.3390/jcm9020538

32. Zhou C. Evaluating new evidence in the early dynamics of the novel coronavirus COVID-19 outbreak in Wuhan, China with real time domestic traffic and potential asymptomatic transmissions. medRxiv [Preprint]. (2020). doi: 10.1101/2020.02.15.20023440

33. World Health Organization. Coronavirus Disease 2019 (COVID-19): Situation Report. WHO (2020).

34. Wu Z, McGoogan JM. Characteristics of and important lessons from the coronavirus disease 2019 (COVID-19) outbreak in China: summary of a report of 72314 cases from the Chinese Center for Disease Control and Prevention. JAMA. (2020) 323:1239-42. doi: 10.1001/jama.2020.2648

35. Kissler S, Tedijanto C, Goldstein E, Grad Y, Lipsitch M. Projecting the transmission dynamics of SARS-CoV-2 through the postpandemic period. Science. (2020) 368:860-8. doi: 10.1101/2020.03.04.20031112

36. Grasselli G, Pesenti A, Cecconi M. Critical care utilization for the COVID19 outbreak in Lombardy, Italy: early experience and forecast during an emergency response. JAMA. (2020) 323:1545-6. doi: 10.1001/jama.2020.4031

37. Zou L, Ruan F, Huang M, Liang L, Huang H, Hong Z, et al. SARS-CoV-2 viral load in upper respiratory specimens of infected patients. New Engl J Med. (2020) 382:1177-9. doi: 10.1056/NEJMc2001737

Conflict of Interest: The authors declare that the research was conducted in the absence of any commercial or financial relationships that could be construed as a potential conflict of interest.

Copyright (C) 2021 Iboi, Richardson, Ruffin, Ingram, Clark, Hawkins, McKinney, Horne, Ponder, Denton, Agusto, Oduro and Akinyemi. This is an open-access article distributed under the terms of the Creative Commons Attribution License (CC BY). The use, distribution or reproduction in other forums is permitted, provided the original author(s) and the copyright owner(s) are credited and that the original publication in this journal is cited, in accordance with accepted academic practice. No use, distribution or reproduction is permitted which does not comply with these terms. 